\title{
IIMA

\section{Nonexistence of global solutions to system of semi-linear fractional evolution equations}

\author{
Medjahed Djilali $^{\mathrm{a}^{*}}$ and Ali Hakem ${ }^{\mathrm{b}}$ \\ a Department of Mathematics, Djillali Liabes University, Sidi-Bel-Abbes-22000, Algeria \\ ${ }^{\mathrm{a}, \mathrm{b}}$ Laboratory ACEDP, Djillali Liabes University, Sidi-Bel-Abbes-22000, Algeria \\ *Corresponding author E-mail: djilalimedjahed@yahoo.fr
}

\section{Article Info}

Keywords: Derivatives in the sense of Caputo, Fractional Laplacian, Fujita's critical exponent, Test function, Weak solution.

2010 AMS: Primary 35A01,

Secondary 35D30, 35R11, 26A33

Received: 19 February 2018

Accepted: 10 May 2018

Available online: 30 September 2018

\begin{abstract}
In this research we are interested to Cauchy problem for system of semi-linear fractional evolution equations.

Some authors were concerned with studying of global existence of solutions for the hyperbolic nonlinear equations with a damping term. Our goal is to extend some results obtained by the authors, by studying the system of semi-linear hyperbolic equations with fractional damping term and fractional Laplacian .

Thanks to the test functions method, we prove the nonexistence of nontrivial global weak solutions to the problem.
\end{abstract}

\section{Introduction}

in this paper we are concerned with the following Cauchy problem:

$$
\begin{cases}u_{t t}+(-\Delta)^{\frac{\beta_{1}}{2}} u+D_{0 \mid t}^{\alpha_{1}} u=f(t, x)|u|^{p_{1}}|v|^{q_{1}}, & (t, x) \in(0,+\infty) \times \mathbb{R}^{N} \\ v_{t t}+(-\Delta)^{\frac{\beta_{2}}{2}} v+D_{0 \mid t}^{\alpha_{2}} v=g(t, x)|u|^{p_{2}}|v|^{q_{2}}, & (t, x) \in(0,+\infty) \times \mathbb{R}^{N}\end{cases}
$$

subjected to the conditions

$$
\begin{aligned}
& u(0, x)=u_{0}(x) \geq 0, \quad u_{t}(0, x)=u_{1}(x) \geq 0, \\
& v(0, x)=v_{0}(x) \geq 0,, \quad v_{t}(0, x)=v_{1}(x) \geq 0, .
\end{aligned}
$$

where $p_{1} \geq 0, q_{2} \geq 0, p_{2}>1, q_{1}>1,0<\alpha_{i}<1 \leq \beta_{i} \leq 2, i=1,2$ are constants. $D_{0 / t}^{\alpha_{i}}$ denotes the derivatives of order $\alpha_{i}$ in the sense of Caputo and $(-\Delta)^{\frac{\beta_{i}}{2}}$ is the fractional power of the $(-\Delta)$.

The integral representation of the fractional Laplacian in the $N$-dimensional space is

$$
(-\Delta)^{\beta / 2} \psi(x)=-c_{N}(\beta) \int_{\mathbb{R}^{N}} \frac{\psi(x+z)-\psi(x)}{|z|^{N+\beta}} d z, \quad \forall x \in \mathbb{R}^{N},
$$

where $c_{N}(\beta)=\Gamma((N+\beta) / 2) /\left(2 \pi^{N / 2+\beta} \Gamma(1-\beta / 2)\right)$, and $\Gamma$ denotes the gamma function ( see [16]).

Note that The fractional Laplacian $\left((-\Delta)^{\beta / 2}\right)$ with $1 \leq \beta \leq 2$ is a pseudo-differential operator defined by:

$$
(-\Delta)^{\beta / 2} u(x)=\mathscr{F}^{-1}\left\{|\zeta|^{\beta} \mathscr{F}(u)(\zeta)\right\}(x) \forall x \in \mathbb{R}^{N},
$$


where $\mathscr{F}$ and $\mathscr{F}^{-1}$ are Fourier transform and inverse Fourier transform, respectively.

The functions $f$ and $g$ are non-negatives and assumed to satisfy the conditions

$$
f(t, x) \geq C_{1} t^{\nu_{1}}|x|^{\mu_{1}}, g(t, x) \geq C_{2} t^{v_{2}}|x|^{\mu_{2}}, \text { where } v_{i} \geq 0, \mu_{i} \geq 0, i=1,2 .
$$

The problem of global existence of solutions for nonlinear hyperbolic equations with a damping term have been studied by many researchers in several contexts (see [4], [8], [9] , [12], [18], [20] ), for example, the following Cauchy problem:

$$
\left\{\begin{array}{l}
u_{t t}-\Delta u+u_{t}=|u|^{p}, \quad(t, x) \in(0, \infty) \times \mathbb{R}^{N} \\
u(0, x)=u_{0}(x), \quad u_{t}(0, x)=u_{1}(x), \quad x \in \mathbb{R}^{N},
\end{array}\right.
$$

Todorova-Yordanov [18] showed that, if $p_{c}<p \leq \frac{n}{n-1}$, then (1.4) admits a unique global solution, and they proved that if $1<p<1+\frac{2}{n}$, then the solution $u$ blows up in a finite time.

Fino-Ibrahim and Wehbe [4] generalized the results of Ogawa-Takeda [12] by proving the blow-up of solutions of (1.4) under weaker assumptions on the initial data and they extended this results to the critical case $p_{c}=1+\frac{2}{n}$.

Qi. Zhang [20] studied the case $1<p<1+\frac{2}{n}$, when $\int u_{i}(x) d x>0, i=0,1$, he proved that global solution of (1.4) does not exist. Therefore, he showed that $p=1+\frac{2}{n}$ belongs to the blow-up case.

A. Hakem [8] treated the same type of (1.4), then he extended this result to the case of a system :

$$
\left\{\begin{array}{l}
u_{t t}+-\Delta u+g(t) u_{t}=|v|^{p}, \quad(t, x) \in(0,+\infty) \times \mathbb{R}^{N} \\
v_{t t}+-\Delta v+f(t) v_{t}=|u|^{q}, \quad(t, x) \in(0,+\infty) \times \mathbb{R}^{N} \\
u(0, x)=u_{0}(x), \quad u_{t}(0, x)=u_{1}(x) \\
v(0, x)=v_{0}(x), \quad v_{t}(0, x)=v_{1}(x),
\end{array}\right.
$$

$g(t)$ and $f(t)$ are functions behaving like $t^{\beta}$ and $t^{\alpha}$, respectively, where $0 \leq \beta, \alpha<1$.

Hakem [8] showed that, if

$$
\frac{N}{2} \leq \frac{1}{p q-1} \max [1-\beta+p(1-\alpha), 1-\alpha+q(1-\beta)]-\max (\alpha, \beta),
$$

then the problem (1.5) has only the trivial solution.

By combining the works of the above authors with those of Kirane et al.[10] and Escobido et al.[2], we were able to prove a nonexistence result to (1.1) in the weak formulation.

\section{Preliminaries}

Let us start by introducing the definitions concerning fractional derivatives in the sense of Caputo and the weak local solution to problem (1.1).

Definition 2.1. Let $0<\alpha<1$ and $\zeta^{\prime} \in L^{1}(0, T)$. The left-sided and respectively right-sided Caputo derivatives of order $\alpha$ for $\zeta$ are defined as:

$$
D_{0 \mid t}^{\alpha} \zeta(t)=\frac{1}{\Gamma(1-\alpha)} \int_{0}^{t} \frac{\zeta^{\prime}(s)}{(t-s)^{\alpha}} d s
$$

and

$$
D_{t \mid T}^{\alpha} \zeta(t)=-\frac{1}{\Gamma(1-\alpha)} \int_{t}^{T} \frac{\zeta^{\prime}(s)}{(s-t)^{\alpha}} d s
$$

where $\Gamma$ denotes the gamma function (see [13] p 79).

Definition 2.2. Let $Q_{T}=(0, T) \times \mathbb{R}^{N}, 0<T<+\infty$.

We say that $(u, v) \in\left(L_{l o c}^{1}\left(Q_{T}\right)\right)^{2}$ is a local weak solution to problem (1.1) on $Q_{T}$,

if $\left(f u^{p_{1}} v^{q_{1}}, g u^{p_{2}} v^{q_{2}}\right) \in\left(L_{l o c}^{1}\left(Q_{T}\right)\right)^{2}$, and it satisfies

$$
\begin{aligned}
\int_{Q_{T}} f|u|^{p_{1}}|v|^{q_{1}} \zeta_{1} d x d t & +\int_{\mathbb{R}^{N}} u_{0}(x) \zeta_{1}(0, x) d x+\int_{\mathbb{R}^{N}} u_{1}(x) \zeta_{1}(0, x) d x-\int_{\mathbb{R}^{N}} u_{0}(x) \zeta_{1}(0, x) d x \\
& =\int_{Q_{T}} u \zeta_{1 t} d x d t+\int_{Q_{T}} u D_{t \mid T}^{\alpha_{1}} \zeta_{1} d x d t+\int_{Q_{T}} u(-\Delta)^{\frac{\beta_{1}}{2}} \zeta_{1} d x d t .
\end{aligned}
$$

and

$$
\begin{aligned}
\int_{Q_{T}} g|u|^{p_{2}}|v|^{q_{2}} \zeta_{2} d x d t & +\int_{\mathbb{R}^{N}} v_{0}(x) \zeta_{2}(0, x) d x+\int_{\mathbb{R}^{N}} v_{1}(x) \zeta_{2}(0, x) d x-\int_{\mathbb{R}^{N}} v_{0}(x) \zeta_{2 t}(0, x) d x \\
& =\int_{Q_{T}} v \zeta_{2 t t} d x d t+\int_{Q_{T}} v D_{t \mid T}^{\alpha_{2}} \zeta_{2} d x d t+\int_{Q_{T}} v(-\Delta)^{\frac{\beta_{2}}{2}} \zeta_{2} d x d t .
\end{aligned}
$$

for all test function $\zeta_{j} \in C_{t, x}^{2,2}\left(Q_{T}\right)$ such as $\zeta_{j} \geq 0$ and $\zeta_{j}(T, x)=\zeta_{j_{t}}(T, x)=\zeta_{j_{t}}(0, x)=0, j=1,2$

(see [3] p 5501). 
Remark 2.3. To get the definition 2.2, we multiplying the first equation in (1.1) by $\zeta_{1}$ and the second equation by $\zeta_{2}$, integrating by parts on $Q_{T}=(0, T) \times \mathbb{R}^{N}$ and using the definition 2.1

The integrals in the above definition are supposed to be convergent.

If in the definition $T=+\infty$, the solution $(u, v)$ is called global.

Now, we recall the following integration by parts formula:

$$
\int_{0}^{T} \phi(t)\left(D_{0 \mid t}^{\alpha} \psi\right)(t) d t=\int_{0}^{T}\left(D_{t \mid T}^{\alpha} \phi\right)(t) \psi(t) d t
$$

( see [17], p 46 ).

\section{Main results}

We now in position to announce our result.

Theorem 3.1. Let $p_{2}>1, q_{1}>1,0<\alpha_{i}<1 \leq \beta_{i} \leq 2, i=1,2$, and

$$
\mathscr{A}:=\frac{\alpha_{1}+\frac{\alpha_{2}}{p_{2}}-\left(1-\frac{1}{p_{2} q_{1}}\right)-\frac{1}{p_{2}}\left(\mu_{2} \frac{\alpha_{1}}{\beta_{1}}+v_{2}\right)-\frac{1}{p_{2} q_{1}}\left(\mu_{1} \frac{\alpha_{2}}{\beta_{2}}+v_{1}\right)}{\frac{\alpha_{1}}{\beta_{1} \tilde{p_{2}}}+\frac{\alpha_{2}}{\beta_{2} p_{2} \tilde{q_{1}}}}
$$

and

$$
\mathscr{B}:=\frac{\alpha_{2}+\frac{\alpha_{1}}{q_{1}}-\left(1-\frac{1}{p_{2} q_{1}}\right)-\frac{1}{q_{1}}\left(\mu_{1} \frac{\alpha_{2}}{\beta_{2}}+v_{1}\right)-\frac{1}{p_{2} q_{1}}\left(\mu_{2} \frac{\alpha_{1}}{\beta_{1}}+v_{2}\right)}{\frac{\alpha_{2}}{\beta_{2} \tilde{q_{1}}}+\frac{\alpha_{1}}{\beta_{1} q_{1} \tilde{p_{2}}}}
$$

where $p_{2} \tilde{p_{2}}=p_{2}+\tilde{p_{2}}, q_{1} \tilde{q_{1}}=q_{1}+\tilde{q_{1}}$,

and the conditions (1.3) are fulfilled.

If

$$
N \leq \max \{\mathscr{A} ; \mathscr{B}\}
$$

then the problem (1.1) admits no nontrivial global weak solutions.

Proof. We notice that, in all steps of proof , $C>0$ is a real positive number which may change from line to line.

Set $\zeta_{j}(t, x)=\Phi\left(\frac{t^{2}+|x|^{2 \theta_{j}}}{R^{2}}\right), j=1,2$ such as $\Phi$ is a decreasing function $C_{0}^{2}\left(\mathbb{R}^{+}\right)$, satisfies

$$
0 \leq \Phi \leq 1 \text { and } \Phi(r)=\left\{\begin{array}{lll}
1 & \text { if } 0 \leq r \leq 1 \\
0 & \text { if } \quad r \geq 2
\end{array}\right.
$$

Where $R>0, \theta_{1}=\beta_{1} / \alpha_{1}$ and $\theta_{2}=\beta_{2} / \alpha_{2}$ (see [10]).

Multiplying the first equation of (1.1) by $\zeta_{1}$ and integrating by parts on $Q_{T}=(0, T) \times \mathbb{R}^{N}$, we get

$$
\begin{aligned}
\int_{Q_{T}} f|u|^{p_{1}}|v|^{q_{1}} \zeta_{1} d x d t & +\int_{\mathbb{R}^{N}} u_{0}(x) \zeta_{1}(0, x) d x+\int_{\mathbb{R}^{N}} u_{1}(x) \zeta_{1}(0, x) d x-\int_{\mathbb{R}^{N}} u_{0}(x) \zeta_{1 t}(0, x) d x \\
& =\int_{Q_{T}} u \zeta_{1 t t} d x d t-\int_{Q_{T}} u D_{0 \mid t}^{\alpha_{1}} \zeta_{1} d x d t+\int_{Q_{T}} u(-\Delta)^{\frac{\beta_{1}}{2}} \zeta_{1} d x d t .
\end{aligned}
$$

It is clear that $\zeta_{j_{t}}(t, x)=2 R^{-2} t \Phi^{\prime}\left(\frac{t^{2}+|x|^{2 \theta_{j}}}{R^{2}}\right)$, consequently $\zeta_{j_{t}}(0, x)=0$, thus

$$
\begin{aligned}
\int_{Q_{T}} f|u|^{p_{1}}|v|^{q_{1}} \zeta_{1} d x d t & +\int_{\mathbb{R}^{N}} u_{0}(x) \zeta_{1}(0, x) d x+\int_{\mathbb{R}^{N}} u_{1}(x) \zeta_{1}(0, x) d x \\
& =\int_{Q_{T}} u \zeta_{1 t t} d x d t+\int_{Q_{T}} u D_{t \mid T}^{\alpha_{1}} \zeta_{1} d x d t+\int_{Q_{T}} u(-\Delta)^{\frac{\beta_{1}}{2}} \zeta_{1} d x d t .
\end{aligned}
$$

Hence,

$$
\int_{Q_{T}} f|u|^{p_{1}}|v|^{q_{1}} \zeta_{1} d x d t \leq \int_{Q_{T}}|u|\left|\zeta_{1 t t}\right| d x d t+\int_{Q_{T}}|u|\left|D_{t \mid T}^{\alpha_{1}} \zeta_{1}\right| d x d t+\int_{Q_{T}}|u|\left|(-\Delta)^{\frac{\beta_{1}}{2}} \zeta_{1}\right| d x d t .
$$

We have also

$$
\int_{Q_{T}} g|u|^{p_{2}}|v|^{q_{2}} \zeta_{2} d x d t \leq \int_{Q_{T}}|v|\left|\zeta_{2 t t}\right| d x d t+\int_{Q_{T}}|v|\left|D_{t \mid T}^{\alpha_{2}} \zeta_{2}\right| d x d t+\int_{Q_{T}}|v|\left|(-\Delta)^{\frac{\beta_{2}}{2}} \zeta_{2}\right| d x d t .
$$


174

Universal Journal of Mathematics and Applications

To estimate

$$
\int_{Q_{T}}|u|\left|\zeta_{t t}\right| d x d t
$$

we observe that it can be rewritten as

$$
\int_{Q_{T}}|u|\left|\zeta_{1 t}\right| d x d t=\int_{Q_{T}}|u|\left(g|v|^{q_{2}} \zeta_{2}\right)^{\frac{1}{p_{2}}}\left|\zeta_{t t}\right|\left(g|v|^{q_{2}} \zeta_{2}\right)^{\frac{-1}{p_{2}}} d x d t .
$$

Using Hölder's inequality, we obtain

$$
\int_{Q_{T}}|u|\left|\zeta_{1_{t t}}\right| d x d t \leq\left(\int_{Q_{T}}|u|^{p_{2}}\left(g|v|^{q_{2}} \zeta_{2}\right) d x d t\right)^{\frac{1}{p_{2}}}\left(\int_{Q_{T}}\left|\zeta_{1_{t t}}\right|^{\frac{p_{2}}{p_{2}-1}}\left(g|v|^{q_{2}} \zeta_{2}\right)^{\frac{-1}{p_{2}-1}} d x d t\right)^{\frac{p_{2}-1}{p_{2}}} .
$$

Proceeding as above, we have

$$
\begin{aligned}
\int_{Q_{T}}|u|\left|D_{t \mid T}^{\alpha_{1}} \zeta_{1}\right| d x d t \leq & \left(\int_{Q_{T}}|u|^{p 2}\left(g|v|^{q_{2}} \zeta_{2}\right) d x d t\right)^{\frac{1}{p_{2}}} \\
& \times\left(\int_{Q_{T}}\left|D_{t \mid T}^{\alpha_{1}} \zeta_{1}\right|^{\frac{p_{2}}{p_{2}-1}}\left(g|v|^{q_{2}} \zeta_{2}\right)^{\frac{-1}{p_{2}-1}} d x d t\right)^{\frac{p_{2}-1}{p_{2}}},
\end{aligned}
$$

and

$$
\begin{aligned}
\int_{Q_{T}}|u|\left|(-\Delta)^{\frac{\beta_{1}}{2}} \zeta_{1}\right| d x d t \leq & \left(\int_{Q_{T}}|u|^{p_{2}}\left(g|v|^{q_{2}} \zeta_{2}\right) d x d t\right)^{\frac{1}{p_{2}}} \\
& \times\left(\int_{Q_{T}}\left|(-\Delta)^{\frac{\beta_{1}}{2}} \zeta_{1}\right|^{\frac{p_{2}}{p_{2}-1}}\left(g|v|^{q_{2}} \zeta_{2}\right)^{\frac{-1}{p_{2}-1}} d x d t\right)^{\frac{p_{2}-1}{p_{2}}} .
\end{aligned}
$$

Finally, we infer

$$
\int_{Q_{T}} f|u|^{p_{1}}|v|^{q_{1}} \zeta_{1} d x d t \leq\left(\int_{Q_{T}}|u|^{p_{2}}\left(g|v|^{q_{2}} \zeta_{2}\right) d x d t\right)^{\frac{1}{p_{2}}} \mathscr{K}_{1}
$$

where

$$
\begin{aligned}
\mathscr{K}_{1}= & \left(\int_{Q_{T}}\left|\zeta_{1 t t}\right|^{\frac{p_{2}}{p_{2}-1}}\left(g|v|^{q_{2}} \zeta_{2}\right)^{\frac{-1}{p_{2}-1}} d x d t\right)^{\frac{p_{2}-1}{p_{2}}}+\left(\int_{Q_{T}}\left|D_{t \mid T}^{\alpha_{1}} \zeta_{1}\right|^{\frac{p_{2}}{p_{2}-1}}\left(g|v|^{q_{2}} \zeta_{2}\right)^{\frac{-1}{p_{2}-1}} d x d t\right)^{\frac{p_{2}-1}{p_{2}}} \\
& +\left(\int_{Q_{T}}\left|(-\Delta)^{\frac{\beta_{1}}{2}} \zeta_{1}\right|^{\frac{p_{2}}{p_{2}-1}}\left(g|v|^{q_{2}} \zeta_{2}\right)^{\frac{-1}{p_{2}-1}} d x d t\right)^{\frac{p_{2}-1}{p_{2}}} .
\end{aligned}
$$

Arguing as above we have likewise

$$
\int_{Q_{T}} g|u|^{p_{2}}|v|^{q_{2}} \zeta_{2} d x d t \leq\left(\int_{Q_{T}}|v|^{q_{1}}\left(f|u|^{p_{1}} \zeta_{1}\right) d x d t\right)^{\frac{1}{q_{1}}} \mathscr{K}_{2},
$$

where

$$
\begin{aligned}
\mathscr{K}_{2}= & \left(\int_{Q_{T}}\left|\zeta_{2 t t}\right|^{\frac{q_{1}}{q_{1}-1}}\left(f|u|^{p_{1}} \zeta_{1}\right)^{\frac{-1}{q_{1}-1}} d x d t\right)^{\frac{q_{1}-1}{q_{1}}}+\left(\int_{Q_{T}}\left|D_{t \mid T}^{\alpha_{2}} \zeta_{2}\right|^{\frac{q_{1}}{q_{1}-1}}\left(f|u|^{p_{1}} \zeta_{1}\right)^{\frac{-1}{q_{1}-1}} d x d t\right)^{\frac{q_{1}-1}{q_{1}}} \\
& +\left(\int_{Q_{T}}\left|(-\Delta)^{\frac{\beta_{2}}{2}} \zeta_{2}\right|^{\frac{q_{1}}{q_{1}-1}}\left(f|u|^{p_{1}} \zeta_{1}\right)^{\frac{-1}{q_{1}-1}} d x d t\right)^{\frac{q_{1}-1}{q_{1}}} .
\end{aligned}
$$

Using inequalities (3.5) and (3.6), it yield

$$
\left(\int_{Q_{T}} f|u|^{p_{1}}|v|^{q_{1}} \zeta_{1} d x d t\right)^{\frac{q_{1} p_{2}-1}{q_{1} p_{2}}} \leq \mathscr{K}_{1} \mathscr{K}_{2}^{\frac{1}{p_{2}}}
$$


Universal Journal of Mathematics and Applications

175

similarly, we get

$$
\left(\int_{Q_{T}} g|u|^{p_{2}}|v|^{q_{2}} \zeta_{2} d x d t\right)^{\frac{q_{1} p_{2}-1}{q_{1} p_{2}}} \leq \mathscr{K}_{2} \mathscr{K}_{1}^{\frac{1}{q_{1}}}
$$

Now, in $\mathscr{K}_{1}$ we consider the scale of variables:

$$
t=\tau R, \quad x=y R^{\frac{\alpha_{1}}{\beta_{1}}},
$$

while in $\mathscr{K}_{2}$ we use:

$$
t=\tau R, \quad x=y R^{\frac{\alpha_{2}}{\beta_{2}}},
$$

and use the fact that

$$
d x d t=R^{\left(\frac{N \alpha_{1}}{\beta_{1}}+1\right)} d y d \tau, \zeta_{i t t}=R^{-2} \zeta_{i \tau \tau}, D_{0 \mid t}^{\alpha_{i}} \zeta_{i t}=R^{-\alpha_{i}} D_{0 \mid \tau R}^{\alpha_{i}} \zeta_{i \tau},
$$

$(-\Delta)_{x}^{\frac{\beta_{i}}{2}} \zeta_{i}=R^{-\alpha_{i}}(-\Delta)_{y}^{\frac{\beta_{i}}{2}} \zeta_{i}, i=1,2$

we arrive at

$$
\left(\int_{Q_{T}} f|u|^{p_{1}}|v|^{q_{1}} \zeta_{1} d x d t\right)^{\frac{q_{1} p_{2}-1}{q_{1} p_{2}}} \leq C\left[R^{\gamma_{1}}+R^{\gamma_{2}}+R^{\gamma_{3}}\right] \times\left[R^{\lambda_{1}}+R^{\lambda_{2}}+R^{\lambda_{3}}\right]^{\frac{1}{p_{2}}},
$$

similarly, we have

$$
\begin{aligned}
& \left(\int_{Q_{T}} g|u|^{p_{2}}|v|^{q_{2}} \zeta_{2} d x d t\right)^{\frac{q_{1} p_{2}-1}{q_{1} p_{2}}} \leq C\left[R^{\lambda_{1}}+R^{\lambda_{2}}+R^{\lambda_{3}}\right] \times\left[R^{\gamma_{1}}+R^{\gamma_{2}}+R^{\gamma_{3}}\right]^{\frac{1}{q_{1}}}, \\
& \text { where }\left\{\begin{array}{l}
\gamma_{1}=\left(\frac{N \alpha_{1}}{\beta_{1}}+1\right)\left(\frac{p_{2}-1}{p_{2}}\right)-2-\left(\frac{\mu_{2} \alpha_{1}}{\beta_{1}}+v_{2}\right) \frac{1}{p_{2}} \\
\gamma_{2}=\left(\frac{N \alpha_{1}}{\beta_{1}}+1\right)\left(\frac{p_{2}-1}{p_{2}}\right)-\alpha_{1}-\left(\frac{\mu_{2} \alpha_{1}}{\beta_{1}}+v_{2}\right) \frac{1}{p_{2}} \\
\gamma_{3}=\left(\frac{N \alpha_{1}}{\beta_{1}}+1\right)\left(\frac{p_{2}-1}{p_{2}}\right)-\alpha_{1}-\left(\frac{\mu_{2} \alpha_{1}}{\beta_{1}}+v_{2}\right) \frac{1}{p_{2}}
\end{array}\right. \\
& \text { and }\left\{\begin{array}{l}
\lambda_{1}=\left(\frac{N \alpha_{2}}{\beta_{2}}+1\right)\left(\frac{q_{1}-1}{q_{1}}\right)-2-\left(\frac{\mu_{1} \alpha_{2}}{\beta_{2}}+v_{1}\right) \frac{1}{q_{1}} \\
\lambda_{2}=\left(\frac{N \alpha_{2}}{\beta_{2}}+1\right)\left(\frac{q_{1}-1}{q_{1}}\right)-\alpha_{2}-\left(\frac{\mu_{1} \alpha_{2}}{\beta_{2}}+v_{1}\right) \frac{1}{q_{1}} \\
\lambda_{3}=\left(\frac{N \alpha_{2}}{\beta_{2}}+1\right)\left(\frac{q_{1}-1}{q_{1}}\right)-\alpha_{2}-\left(\frac{\mu_{1} \alpha_{2}}{\beta_{2}}+v_{1}\right) \frac{1}{q_{1}}
\end{array}\right. \\
& \left(\int_{Q_{T}} f|u|^{p_{1}}|v|^{q_{1}} \zeta_{1} d x d t\right)^{\frac{q_{1} p_{2}-1}{q_{1} p_{2}}} \leq C R^{\gamma_{2}+\frac{\lambda_{2}}{p_{2}}}
\end{aligned}
$$

and

$$
\left(\int_{Q_{T}} g|u|^{p_{2}}|v|^{q_{2}} \zeta_{2} d x d t\right)^{\frac{q_{1} p_{2}-1}{q_{1} p_{2}}} \leq C R^{\lambda_{2}+\frac{\gamma_{2}}{q_{1}}}
$$

with the fact that

$$
\frac{1}{p_{2}}+\frac{1}{\tilde{p}_{2}}=1 \text { and } \frac{1}{q_{1}}+\frac{1}{\tilde{q}_{1}}=1
$$

by a simple computation,

$$
\gamma_{2}+\frac{\lambda_{2}}{p_{2}}=N\left(\frac{\alpha_{1}}{\beta_{1} \tilde{p_{2}}}+\frac{\alpha_{2}}{\beta_{2} p_{2} \tilde{q_{1}}}\right)-\left(\alpha_{1}+\frac{\alpha_{2}}{p_{2}}\right)+\frac{1}{\tilde{p_{2}}}+\frac{1}{p_{2} \tilde{q_{1}}}+\frac{1}{p_{2}}\left(\mu_{2} \frac{\alpha_{1}}{\beta_{1}}+v_{2}\right)+\frac{1}{p_{2} q_{1}}\left(\mu_{1} \frac{\alpha_{2}}{\beta_{2}}+v_{1}\right)
$$

and

$$
\lambda_{2}+\frac{\gamma_{2}}{q_{1}}=N\left(\frac{\alpha_{2}}{\beta_{2} \tilde{q_{1}}}+\frac{\alpha_{1}}{\beta_{1} q_{1} \tilde{p_{2}}}\right)-\left(\alpha_{2}+\frac{\alpha_{1}}{q_{1}}\right)+\frac{1}{\tilde{q_{1}}}+\frac{1}{q_{1} \tilde{p_{2}}}+\frac{1}{q_{1}}\left(\mu_{1} \frac{\alpha_{2}}{\beta_{2}}+v_{1}\right)+\frac{1}{p_{2} q_{1}}\left(\mu_{2} \frac{\alpha_{1}}{\beta_{1}}+v_{2}\right)
$$


also, using (3.13) we have

$$
\frac{1}{\tilde{p_{2}}}+\frac{1}{p_{2} \tilde{q_{1}}}=1-\frac{1}{p_{2}}+\frac{1}{p_{2} \tilde{q_{1}}}=1-\frac{1}{p_{2}}\left(1-\frac{1}{\tilde{q_{1}}}\right)=1-\frac{1}{p_{2} q_{1}}
$$

and

$$
\frac{1}{\tilde{q_{1}}}+\frac{1}{q_{1} \tilde{p_{2}}}=1-\frac{1}{q_{1}}+\frac{1}{q_{1} \tilde{p_{2}}}=1-\frac{1}{q_{1}}\left(1-\frac{1}{\tilde{p_{2}}}\right)=1-\frac{1}{p_{2} q_{1}}
$$

we obtain

$$
\gamma_{2}+\frac{\lambda_{2}}{p_{2}}=N\left(\frac{\alpha_{1}}{\beta_{1} \tilde{p_{2}}}+\frac{\alpha_{2}}{\beta_{2} p_{2} \tilde{q_{1}}}\right)-\left(\alpha_{1}+\frac{\alpha_{2}}{p_{2}}\right)+1-\frac{1}{p_{2} q_{1}}+\frac{1}{p_{2}}\left(\mu_{2} \frac{\alpha_{1}}{\beta_{1}}+v_{2}\right)+\frac{1}{p_{2} q_{1}}\left(\mu_{1} \frac{\alpha_{2}}{\beta_{2}}+v_{1}\right)
$$

and

$$
\lambda_{2}+\frac{\gamma_{2}}{q_{1}}=N\left(\frac{\alpha_{2}}{\beta_{2} \tilde{q_{1}}}+\frac{\alpha_{1}}{\beta_{1} q_{1} \tilde{p_{2}}}\right)-\left(\alpha_{2}+\frac{\alpha_{1}}{q_{1}}\right)+1-\frac{1}{p_{2} q_{1}}+\frac{1}{q_{1}}\left(\mu_{1} \frac{\alpha_{2}}{\beta_{2}}+v_{1}\right)+\frac{1}{p_{2} q_{1}}\left(\mu_{2} \frac{\alpha_{1}}{\beta_{1}}+v_{2}\right)
$$

We conclude that

- If $\gamma_{2}+\frac{\lambda_{2}}{p_{2}}<0$, it yield

$$
N<\frac{\alpha_{1}+\frac{\alpha_{2}}{p_{2}}-\left(1-\frac{1}{p_{2} q_{1}}\right)-\frac{1}{p_{2}}\left(\mu_{2} \frac{\alpha_{1}}{\beta_{1}}+v_{2}\right)-\frac{1}{p_{2} q_{1}}\left(\mu_{1} \frac{\alpha_{2}}{\beta_{2}}+v_{1}\right)}{\frac{\alpha_{1}}{\beta_{1} \tilde{p_{2}}}+\frac{\alpha_{2}}{\beta_{2} p_{2} \tilde{q_{1}}}} .
$$

Then the right hand side of (3.11) goes to 0 , when $R$ tends to infinity, while the left hand side converge to

$$
\left(\int_{Q_{T}} f|u|^{p_{1}}|v|^{q_{1}} d x d t\right)^{\frac{q_{1} p_{2}-1}{q_{1} p_{2}}}
$$

This implies that $v \equiv 0$ or $u \equiv 0$.

Similarly, if $\lambda_{2}+\frac{\gamma_{2}}{q_{1}}<0$, it yield

$$
N<\frac{\alpha_{2}+\frac{\alpha_{1}}{q_{1}}-\left(1-\frac{1}{p_{2} q_{1}}\right)-\frac{1}{q_{1}}\left(\mu_{1} \frac{\alpha_{2}}{\beta_{2}}+v_{1}\right)-\frac{1}{p_{2} q_{1}}\left(\mu_{2} \frac{\alpha_{1}}{\beta_{1}}+v_{2}\right)}{\frac{\alpha_{2}}{\beta_{2} \tilde{q_{1}}}+\frac{\alpha_{1}}{\beta_{1} q_{1} \tilde{p_{2}}}},
$$

by using also (3.12) to proceeding as above, we obtain $u \equiv 0$ or $v \equiv 0$.

- If $\gamma_{2}+\frac{\lambda_{2}}{p_{2}}=0$, we get

$$
\int_{\mathbb{R}^{+} \times \mathbb{R}^{N}} f|u|^{p_{1}}|v|^{q_{1}} d x d t<+\infty .
$$

Using again Hölder's inequality, we obtain

$$
\int_{Q_{T}} g|u|^{p_{2}}|v|^{q_{2}} \zeta_{2} d x d t \leq\left(\int_{B_{R}}|v|^{q_{1}}\left(f|u|^{p_{1}} \zeta_{1}\right) d x d t\right)^{\frac{1}{q_{1}}} \mathscr{K}_{2},
$$

where

$$
B_{R}=\left\{(t, x) \in \mathbb{R}^{+} \times \mathbb{R}^{N} ; R^{2} \leq t^{2}+|x|^{2 \theta_{1}} \leq 2 R^{2}\right\} .
$$

Since,

$$
\int_{\mathbb{R}^{+} \times \mathbb{R}^{N}} f|u|^{p_{1}}|v|^{q_{1}} d x d t<+\infty
$$

we get

$$
\lim _{R \rightarrow+\infty} \int_{B_{R}} f|u|^{p_{1}}|v|^{q_{1}} d x d t=0
$$

hence, we infer that

$$
\int_{\mathbb{R}^{+} \times \mathbb{R}^{N}} g|u|^{p_{2}}|v|^{q_{2}} d x d t=0
$$

this implies that $v \equiv 0$ or $u \equiv 0$.

Similarly, if $\lambda_{2}+\frac{\gamma_{2}}{q_{1}}=0$, proceeding as above, we infer that $u \equiv 0$ or $v \equiv 0$.

We deduce that no global weak solution is possible other than the trivial one, which ends the proof.

Remark 3.2. In the case $\alpha_{i}=1, \beta_{i}=2, v_{i}=\mu_{i}=0, p_{1}=q_{2}=0$,

$i=1,2$, we recover the case who studied by A. Hakem (see [8]), when $\alpha=\beta=0$. 


\section{Acknowledgments}

The authors would like to express their gratitude to the referee. We are very grateful for his helpful comments and careful reading, which have led to the improvement of the manuscript.

\section{References}

[1] M. BERBICHE, A. HAKEM, Necessary conditions for the existence and sufficient conditions for the nonexistence of solutions to a certain fractional telegraph equation. Memoirs on Differential Equations and Mathematical physics. vol 56, 2012, 37-55.

[2] M. Escobedo \& H. A. LEVINE, Critical blow up and global existence numbers of a weakly coupled system of reaction-diffusion equation. Arch. Rational. Mech. Anal. 129 (1995),47-100.

[3] A. Z. Fino, Critical exponent for damped wave equations with nonlinear memory. Nonlinear Analysis 74 (2011) $5495-5505$.

[4] A. Z. FINO, H. IBRAHIM \& A. WEHBE, A blow-up result for a nonlinear damped wave equation in exterior domain: The critical case, Computers \& Mathematics with Applications, Volume 73, Issue 11, (2017), pp. 2415-2420.

[5] H. FujitA, On the blowing up of solutions of the problem for $u_{t}=\Delta u+u^{1+\alpha}$, J. Fac. Sci.Univ. Tokyo 13 (1966), $109-124$

[6] M. GUEDDA \& M. KIRANE, Local and global nonexistence of solutions to semilinear evolution equations. Electronic Journal of Differential Equations, Conference 09 (2002), pp. 149-160.

[7] B. Guo, X. Pu \& F. HuAng, Fractional Partial Differential Equations and Their Numerical Solutions. World Scientific Publishing Co. Pte. Ltd. Beijing, China (2011).

[8] A. HAKEM, Nonexistence of weak solutions for evolution problems on $\mathbb{R}^{N}$, Bull. Belg. Math. Soc. 12 (2005), 73-82.

[9] A. HAKEM \& M. BERBICHE, On the blow-up of solutions to semi-linear wave models with fractional damping . IAENG International Journal of Applied Mathematics, (2011) 41:3, IJAM-41-3-05.

[10] M. KIRANE, Y. LASKRI \& N.-E.TATAR, Critical exponents of fujita type for certain evolution equations and systems with spation-temporal fractional derivatives.J. Math. Anal. Appl. 312 (2005) 488-501.

[11] W. MingXIN, Global existence and finite time blow up for a reaction-diffusion system. Z. Angew. Math. Phys. 51 (2000) $160-167$.

[12] T. OGAWA \& H. TAKIDA, Non-existence of weak solutions to nonlinear damped wave equations in exterior domains, J. Nonliniear analysis 70 (2009), 3696-3701.

[13] I. PodLubny, Fractional differential equations. Mathematics in Science and Engineering, vol 198, Academic Press, New York, 1999.

[14] S.I. Pohozaev \& A. TeSEI, Nonexistence of Local Solutions to Semilinear Partial Differential Inequalities, Nota Scientifica 01/28, Dip. Mat. Universitá "La Sapienza", Roma (2001).

[15] S. Pohozaty \& L. Veron, Blow up results for nonlinear hyperbolic enequalities, Ann. Scuola Norm. Sup. Pisa CI. Sci. (4) Vol. XXIX (2000), pp. $393-420$.

[16] C. POZRIKIDIS, The fractional Laplacian, Taylor \& Francis Group, LLC /CRC Press, Boca Raton (USA), (2016).

[17] S. G. SAmkO, A. A. KIlbAS \& O. I. MARICHEV, Fractional integrals and derivatives: Theory and applications. Gordan and Breach Sci. Publishers, Yverdon, 1993

[18] G.TODOROVA \& B.YordANOv, Critical Exponent for a Nonlinear Wave Equation with Damping. Journal of Differential Equations 174, 464-489 (2001)

[19] Y. YAMAUCHI, Blow-up results for a reaction-deffusion system, Methods Appl. Anal. 13 (2006), 337 - 350

[20] Q. S. ZHANG, A blow up result for a nonlinear wave equation with damping: the critical case, C. R. Acad.Sci. paris, Volume 333, no.2, (2001), 109-114.

[21] S-MU. ZHENG, Nonlinear evolution equations, Chapman \& Hall/CRC Press, Florida (USA), (2004). 